ORIGINAL PAPER

\title{
The CONCORDANCE OF DNA MISMATCH REPAIR PROTEIN \\ BETWEEN ENDOSCOPIC BIOPSIES AND SURGICAL SPECIMENS \\ AND INTER-OBSERVERS VARIATIONS IN COLORECTAL CANCER \\ PATIENTS: REFLECTIONS FROM ENDOSCOPE DOCTORS
}

\author{
Chunmei Guo ${ }^{1,2}$, Shutian Zhang ${ }^{1}$, Jing Wu ${ }^{1,2}$
}

${ }^{1}$ Department of Gastroenterology, Beijing Friendship Hospital, Capital Medical University, Xicheng District, Beijing, China

${ }^{2}$ Department of Gastroenterology, Beijing Shijitan Hospital, Capital Medical University, Haidian District, Beijing, China

\begin{abstract}
Previous studies showed that the yield of immunohistochemistry (IHC) staining on endoscopic biopsies may be as good as on surgically removed tissues. However, we noted that some patients showed inconsistent DNA mismatch repair protein (MMRP) stains between biopsies and surgical specimens. In this study, we aimed to investigate factors which are related to the consistence of MMRP evaluation between two pathologists or between different tissues. Two pathologists were asked to diagnose 4 MMRP, both on endoscopic biopsies and surgical materials, in 51 colorectal cancer (CRC) patients, using a single blind method. The consistence of two specimens and inter-observers' variances across different pathologists were compared respectively and the factors related to this variability were analyzed. Among the 816 paired MMRP, 804 (98.5\%) pairs showed concordant IHC stains between biopsies and surgical materials, the agreement was almost perfect for MSH6 and PMS2 ( $\kappa=0.85,0.85$ separately); 804 (98.5\%) pairs showed concordant IHC stains between two pathologists, the inter-observer agreement was almost perfect for MSH6 and PMS2 ( $\kappa=0.85,0.88$ separately). Clinical and pathological characteristics analysis showed that biopsy number and TNM stage were related to the variations. Inter-observers variations should be taken into account during MMRP testing in colorectal cancers. Generous endoscopic biopsies could improve the accuracy of endoscopic biopsy for MMRP detection which can be used histological tool in the evaluation of CRC and a promising new prognostic factor for these patients.
\end{abstract}

Key words: colorectal cancer, endoscopic biopsy, surgical specimens, DNA mismatch repair protein.

\section{Introduction}

Colorectal cancer (CRC) is the third leading cause of cancer-related deaths around the world [1]. Every year, 1,000,000 new CRC cases and 700,000 mortalities caused by CRC are registered [2]. Cumulative genetics and epigenetic alterations, such as DNA mismatch repair (MMR) and cell cycle regulators' genes participate in the development of CRC [3]. MMR deficient (dMMR), mainly due to the negative expression of mismatch repair proteins (MLH1, MSH2, PMS1 and/or PMS2) [4], associates with 
distinct clinicopathological features of CRC, including poorly differentiated tumor cells, presence of mucin, lymphocytic infiltrate and predominantly proximal colon cancer. Patients with dMMR may reflect better overall survival rate and better response to 5-FU based therapies [5].

In recent guidelines, it was suggested that all CRC patients should be tested 4 MMRP through immunohistochemistry (IHC) staining [6, 7]. Previous studies have shown that the yield of IHC staining on endoscopic biopsies may be as good as on surgically resected tissues $[8,9,10,11]$. Biopsy samples may provide superior staining with a faster and more thorough fixation, given the sensitivity of immunostaining associates with the degree of tissue fixation. However, we noted that IHC staining for MMRP on endoscopic biopsies was not entirely consistent with the same staining on resection specimens in some patients [12]. As the first step for further genetic evaluation, proper interpretation of IHC staining will have a profound impact on selected treatment strategy [13]. So it is very important to ensure the accuracy of MMRP detection on endoscopic biopsies, especially for terminal stage CRC that can't be resected on. In this study, we ask two pathologists to diagnose 4 MMRP both on endoscopic biopsies and surgical materials in CRC patients, record the consistence between two specimens and inter-observers' variations respectively and analyze the factors related to the variations.

\section{Material and methods}

\section{Study design}

Between March 2013 to September 2017, CRC patients both taken endoscopy biopsy and surgical resection were consecutively recruited. Patients were included if the following criteria were fulfilled: 1) primary colorectal cancer diagnosed by pathology; 2) both endoscopic biopsy and radical resection of colorectal cancer were performed, and MMRP was detected in both the biopsy and surgical specimens; 3) no chemotherapy, radiotherapy or other antitumor regimens before the operation. Patients' demographic features, clinical details and histological parameters of the tumors were recorded. The MMRP (MLH1, MSH2, MSH6 and PMS2) of endoscopic/surgical specimens was assigned to two senior pathologists by random distribution with single blind method, that means the pathologists didn't know the pairing of the specimens in advance. The inter-observers' variation analysis was carried out by the researcher.

\section{Immunohistochemistry staining for MMRP}

MMRP, included MLH1, MSH2, MSH6 and PMS2 performed on both preoperative endoscopic biopsies and subsequent surgical resection specimens were analyzed. Thin $(5 \mu \mathrm{m})$ sections of representative blocks were taken from the areas with colon tumor cells $>50 \%$. Following antigen retrieval in repair solution EnVisiona (Dako, Denmark), the mouse monoclonal antibodies against MLH1, MSH2, MSH6 and PMS2 (Dako Company, Denmark) were added, incubated at room temperature for $20 \mathrm{~min}$, phosphate buffered saline (PBS) rinsed 3 times for $5 \mathrm{~min}$. Incubations were performed with a secondary antibody, followed by staining (Diaminobenzidine as a chromogen) and nuclear counterstain (by hematoxylin). Phosphate buffered saline (PBS) was used as negative control and normal colorectal mucosa epithelium as positive control. Interpretation of the stains was performed according to the methods described by Shia et al. [13] and Barrow et al. [14]: "positive expression" showed the overall nuclear staining in tumor cells and internal non-neoplastic cells; "negative expression" was defined as the epithelial cells within the tumor tissue lacked nuclear staining while the surrounding stromal cells were positive for MMR staining; staining in less than $10 \%$ of the tumor with only a weak or moderate intensity was described as "focal positive expression". pMMR was used to describe tumor tissue with all MMRP present, while dMMR represents those showing deficiency in at least one of the 4 MMRP [12].

\section{Statistical analysis}

The agreement between endoscopic biopsies and surgical resections or inter-observers' variation were evaluated by using ICC (0-0.2: slight reliability, $0.2-$ 0.4: fair reliability, 0.4-0.6: moderate reliability, 0.60.8 : substantial reliability, $>0.8$ : almost perfect reliability) [15]. Factors associated with the consistence between specimens in detecting MMRP were analyzed by constructing a 2-2 matrix (consistent group vs inconsistent group). Two-sided p-values of $<0.05$ were considered as significant. Statistical analyses were performed with IBM SPSS Statistics 20.0 (SPSS Inc, Chicago, Ill).

\section{Results}

\section{Patient demographics}

Fifty-one patients with CRC were included as the training cohort. Mean patient age was 66.4 years, with an age range of 43-94 years. Twenty-four $(47.1 \%)$ patients were female, and $27(52.9 \%)$ were male. Tumor locations were as follows: proximal colon $-19(37.3 \%)$, distal colon $-17(33.3 \%)$, rectum $15(29.4 \%)$ (Table I). 
Table I. Patients' demographic features, clinical details and histological parameters of the tumors

\begin{tabular}{|c|c|c|}
\hline Parameter & StRATA & $\begin{array}{l}\text { TrAINING } \\
\text { COHORT } \\
(\mathrm{N}=51)\end{array}$ \\
\hline Mean age (years) $\pm S D$ & 23.08 & $66.4(10.5)$ \\
\hline (Range) & 46.15 & $43-94$ \\
\hline \multirow[t]{2}{*}{ Gender, n (\%) } & Female & $24(47.1)$ \\
\hline & Male & $27(52.9)$ \\
\hline \multirow[t]{3}{*}{ Tumor site, n (\%) } & Proximal & $19(37.3)$ \\
\hline & Distal & $17(33.3)$ \\
\hline & Rectum & $15(29.4)$ \\
\hline \multirow[t]{3}{*}{ Tumor size, n (\%) } & $\leq 2 \mathrm{~cm}$ & $5(9.8)$ \\
\hline & $2-5 \mathrm{~cm}$ & $17(33.3)$ \\
\hline & $>5 \mathrm{~cm}$ & $29(56.9)$ \\
\hline \multirow[t]{2}{*}{ TNM, n (\%) } & I-II & $19(37.3)$ \\
\hline & III-IV & $32(62.7)$ \\
\hline \multirow[t]{2}{*}{ LVI, n (\%) } & Absent & $27(52.9)$ \\
\hline & Present & $24(47.1)$ \\
\hline \multirow[t]{2}{*}{ EMVI, n (\%) } & Absent & $34(66.7)$ \\
\hline & Present & $17(33.3)$ \\
\hline \multirow[t]{2}{*}{ Margin, n (\%) } & Expansile & $44(86.3)$ \\
\hline & Infiltrative & $7(13.7)$ \\
\hline
\end{tabular}

The consistence between two specimens for each MMRP

To observe the consistency between endoscopic biopsies and surgical specimens, it was found that among the 816 paired MMRP, 804 (98.5\%) pairs showed concordant endoscopic and operative material stains, either positive $(788,96.6 \%)$, focal positive $(6,0.7 \%)$ or negative $(10,1.2 \%)$. The agreement was almost perfect for MSH6 and PMS2 $(\kappa=0.85$, 0.85 separately), substantial for MLH1 and MSH2 $(\kappa=0.75,0.66$ separately; Table II).

\section{Inter-observers' variability for each MMRP}

Among the $816(102 \times 4)$ paired MMRP, 804 (98.5\%) pairs showed concordant IHC stains between two pathologists, either positive (790, $98.3 \%)$, focal positive $(4,0.5 \%)$ or negative (10, $1.2 \%)$. The inter-observers' agreement was almost perfect for MSH6 and PMS2 $(\kappa=0.85,0.88$ separately), substantial for MLH1 and $\mathrm{MSH} 2(\kappa=$ $0.74,0.71$ separately; Table III). Specimens with intra-observer variability were collected from 5 patients. The patients' demographics, MMRP status in endoscopic biopsies and resected specimens, MMRP status diagnosed by two pathologists were shown in Table IV.

Table II. Agreement between biopsy and surgical material for all MMRP

\begin{tabular}{|c|c|c|c|c|c|c|}
\hline \multirow[t]{2}{*}{ MMRP } & \multirow{2}{*}{$\begin{array}{l}\text { ENDOSCOPY } \\
\text { BIOPSY }\end{array}$} & \multicolumn{3}{|c|}{ SURGICAL MATERIAL } & \multirow[t]{2}{*}{ Total } & \multirow[t]{2}{*}{$\mathrm{K}$} \\
\hline & & Negative & FOCAL & Positive & & \\
\hline \multirow[t]{4}{*}{ MLH1 } & Negative & 2 & 0 & 2 & 4 & 0.75 (substantial) \\
\hline & Focal & 0 & 2 & 0 & 2 & \\
\hline & Positive & 0 & 0 & 198 & 198 & \\
\hline & Total & 2 & 2 & 200 & 204 & \\
\hline \multirow[t]{4}{*}{ MSH2 } & Negative & 2 & 0 & 0 & 2 & 0.66 (substantial) \\
\hline & Focal & 1 & 2 & 0 & 2 & \\
\hline & Positive & 1 & 0 & 198 & 200 & \\
\hline & Total & 4 & 2 & 200 & 204 & \\
\hline \multirow[t]{4}{*}{ MSH6 } & Negative & 2 & 2 & 0 & 4 & 0.85 (almost perfect) \\
\hline & Focal & 0 & 0 & 0 & 0 & \\
\hline & Positive & 0 & 0 & 200 & 200 & \\
\hline & Total & 2 & 2 & 200 & 204 & \\
\hline \multirow[t]{4}{*}{ PMS2 } & Negative & 4 & 2 & 0 & 6 & 0.85 (almost perfect) \\
\hline & Focal & 0 & 2 & 0 & 2 & \\
\hline & Positive & 1 & 0 & 195 & 196 & \\
\hline & Total & 5 & 4 & 195 & 204 & \\
\hline
\end{tabular}


Table III. Inter-observer variability for all MMRP

\begin{tabular}{|c|c|c|c|c|c|c|}
\hline \multirow[t]{2}{*}{ MMRP } & \multirow[t]{2}{*}{ Pathologist A } & \multicolumn{3}{|c|}{ Pathologist B } & \multirow[t]{2}{*}{ Total } & \multirow[t]{2}{*}{$\mathrm{K}$} \\
\hline & & Negative & FOCAL & Positive & & \\
\hline \multirow[t]{4}{*}{ MLH1 } & Negative & 2 & 0 & 0 & 2 & 0.74 (substantial) \\
\hline & Focal & 0 & 2 & 0 & 2 & \\
\hline & Positive & 2 & 0 & 198 & 200 & \\
\hline & Total & 4 & 2 & 198 & 204 & \\
\hline \multirow[t]{4}{*}{ MSH2 } & Negative & 2 & 0 & 0 & 2 & 0.71 (substantial) \\
\hline & Focal & 1 & 0 & 2 & 2 & \\
\hline & Positive & 0 & 1 & 198 & 200 & \\
\hline & Total & 4 & 0 & 200 & 204 & \\
\hline \multirow[t]{4}{*}{ MSH6 } & Negative & 2 & 2 & 0 & 4 & 0.85 (almost perfect) \\
\hline & Focal & 0 & 0 & 0 & 0 & \\
\hline & Positive & 0 & 0 & 200 & 200 & \\
\hline & Total & 2 & 2 & 200 & 204 & \\
\hline \multirow[t]{4}{*}{ PMS2 } & Negative & 4 & 2 & 0 & 6 & 0.88 (almost perfect) \\
\hline & Focal & 0 & 2 & 0 & 2 & \\
\hline & Positive & 0 & 1 & 195 & 196 & \\
\hline & Total & 4 & 5 & 195 & 204 & \\
\hline
\end{tabular}

Table IV. The 5 patients' demographics, MMRP status in endoscopic biopsies and resected specimens, MMRP status diagnosed by two pathologists

\begin{tabular}{|c|c|c|c|c|c|c|c|c|c|}
\hline No. & AgE & SeX & $\begin{array}{l}\text { Tumour } \\
\text { SITE }\end{array}$ & $\begin{array}{l}\text { Tumour } \\
\text { SIZE }\end{array}$ & $\begin{array}{l}\text { TNM } \\
\text { STAGE }\end{array}$ & $\begin{array}{l}\text { MMRP } \\
\text { NEGAtive } \\
\text { (bx) or } \\
\text { focal }\end{array}$ & $\begin{array}{l}\text { MMRP } \\
\text { NEGAtive } \\
\text { (rs) or } \\
\text { focal }\end{array}$ & $\begin{array}{c}\text { PATHOLOGIst } \\
\text { A }\end{array}$ & $\begin{array}{c}\text { Pathologist } \\
\text { B }\end{array}$ \\
\hline 1 & 82 & M & $\mathrm{HF}$ & 2 & I & $\begin{array}{l}\text { MLH1, } \\
\text { PMS2 }\end{array}$ & MSH2 & MSH2 & $\begin{array}{l}\text { MlH1, } \\
\text { PMS2 }\end{array}$ \\
\hline 2 & 72 & $\mathrm{~F}$ & Caecum & 6 & II & $\begin{array}{l}\text { MLH1, } \\
\text { MSH2* }\end{array}$ & MSH2 & $\mathrm{MSH} 2 *$ & $\begin{array}{l}\text { MlH1, } \\
\text { MSH2 }\end{array}$ \\
\hline 3 & 76 & M & Rectum & 6 & II & PMS2 & PMS2* & PMS2 & PMS2* \\
\hline 4 & 59 & $\mathrm{~F}$ & Rectum & 4 & II & MSH6 & MSH6* & MSH6 & MSH6* \\
\hline 5 & 61 & $\mathrm{M}$ & Descend & 10 & IV & $\begin{array}{l}\text { MSH6, } \\
\text { PMS2 }\end{array}$ & $\begin{array}{l}\text { MSH6* } \\
\text { PMS2* }\end{array}$ & $\begin{array}{c}\text { MSH6, } \\
\text { PMS2 }\end{array}$ & $\begin{array}{l}\text { MSH6* } \\
\text { PMS2* }\end{array}$ \\
\hline
\end{tabular}

bx - biopsy; MMRP - mismatch repair protein; Mut-mutated; $r$ - resection specimen; HF - bepatic flexure; * focal positive

\section{Factors associated with inter-observers' variability}

According to the consistency of MMRP between endoscopic biopsy and operative specimens or between two pathologists, 51 patients were divided into two groups: consistent group (46 patients) which was defined as a consistent MMRP result between two specimens, while inconsistent group (5 patients) was described as inconsistent MMRP results. Clinical and pathological characteristics analysis showed that more patients in the consistent group had over $4 \mathrm{bi}-$ opsies taken than those in the inconsistent group $(76.1 \%$ vs. $20.0 \%, p=0.036$ ). The accuracy of endoscopic biopsy specimens increased among patients with TNM stage III-IV CRC than that with stage I-II $(78.3 \%$ vs. $20.0 \%, \mathrm{p}=0.025$; Table V).

\section{Discussion}

Colorectal cancer (CRC) is a heterogeneous group of diseases both from the morphological and molecular point of view [16]. As far as we know, CRC is a heterogeneous clinical entity characterized by multiple molecular subtypes. Two main subtypes are 'classic' chromosomal instability pathway and microsatellite 
Table V. Comparison of histopathological features according to mismatch repair protein status consistence in colorectal cancer

\begin{tabular}{|c|c|c|c|c|c|}
\hline Parameter & Strata & $\mathbf{N}$ & $\begin{array}{c}\text { CONSISTENT } \\
\text { GROUP } \\
(\mathrm{N}=46)\end{array}$ & $\begin{array}{c}\text { INCONSISTENT } \\
\text { GROUP } \\
(\mathrm{N}=5)\end{array}$ & P-VALUe \\
\hline Mean age (years) $\pm S D$ & & 51 & $66.3(10.6)$ & $67.0(10.5)$ & $0.9 \#$ \\
\hline (Range) & & 51 & 43-94 & $52-82$ & \\
\hline \multirow[t]{2}{*}{ Gender, n (\%) } & Female & 24 & $22(47.8)$ & $2(40.0)$ & 1.0 \\
\hline & Male & 27 & $24(52.1)$ & $3(60.0)$ & \\
\hline \multirow[t]{3}{*}{ Site, n (\%) } & Proximal & 19 & $17(37.0)$ & $2(40.0)$ & $0.851 *$ \\
\hline & Distal & 17 & $16(34.8)$ & $1(20.0)$ & \\
\hline & Rectum & 15 & $13(28.3)$ & $2(40.0)$ & \\
\hline \multirow[t]{3}{*}{ Size, n (\%) } & $\leq 2 \mathrm{~cm}$ & 5 & $4(8.7)$ & $1(20.0)$ & $0.617^{*}$ \\
\hline & $2-5 \mathrm{~cm}$ & 17 & $16(34.8)$ & $1(20.0)$ & \\
\hline & $>5 \mathrm{~cm}$ & 29 & $26(56.5)$ & $3(60.0)$ & \\
\hline \multirow[t]{2}{*}{ Biopsy number, n (\%) } & $\leq 4$ & 15 & $11(23.9)$ & $4(80.0)$ & 0.036 \\
\hline & $>4$ & 36 & $35(76.1)$ & $1(20.0)$ & \\
\hline \multirow[t]{2}{*}{ TNM, n (\%) } & I-II & 14 & $10(21.7)$ & $4(80.0)$ & 0.025 \\
\hline & III-IV & 37 & $36(78.3)$ & $1(20.0)$ & \\
\hline \multirow[t]{2}{*}{ LVI, n (\%) } & Absent & 27 & $24(52.2)$ & $3(60.0)$ & 1.0 \\
\hline & Present & 24 & $22(47.8)$ & $2(40.0)$ & \\
\hline \multirow[t]{2}{*}{$\mathrm{EMV}, \mathrm{n}(\%)$} & Absent & 34 & $32(69.6)$ & $2(40.0)$ & 0.405 \\
\hline & Present & 17 & $14(30.4)$ & $3(60.0)$ & \\
\hline \multirow[t]{2}{*}{ Margin, n (\%) } & Expansile & 44 & $41(89.1)$ & $3(60.0)$ & $0.133^{*}$ \\
\hline & Infiltrative & 7 & $5(10.9)$ & $2(40.0)$ & \\
\hline
\end{tabular}

\#Independent samples Student's t-test; *Fisher's exact test; TNM - tumor-node-metastasis; LVI - lymphovascular invasion; EMVI - extramural venous invasion

instability pathway (MSI). Microsatellite instability is reflective of a deficient mismatch repair system (dMMR), which may be due to either sporadic or germline mutations in the relevant mismatch repair (MMR) gene [17]. In China, IHC staining detection of MMRP was used in most laboratories to represent MSI owing to its simplicity and veracity $[18]$.

According to MMR status, CRC was divided into two distinct types: MMR proficient (pMMR) defined as positive expression of all MMRP (MLH1, MSH2, MSH6 and PMS2) while MMR deficient (dMMR) characterized by one or more negative expression of MMRP (mostly MLH1 or MSH2, rarely MSH6 or PMS2) [14, 15]. dMMR is observed in 10-20\% CRC patients [19] with several distinct clinicopathological features, such as predominantly proximal colon cancer, poorly differentiated, mucin tumor cells and lymphocytic infiltrate. Due to its influence in CRC chemotherapy, dMMR was served as a predictive factor to 5 -FU based treatment in CRC patients [20, 21].

MMR status is frequently determined by IHC for MMRP on surgical specimens [10]. A number of studies have shown that the yield of IHC staining performed on endoscopic biopsies may be as good as operation materials [8]. Vilkin et al. found that from a procedural perspective given the sensitivity of immunostaining to the degree of tissue fixation, endoscopic biopsy material may produce superior staining based on faster and more thorough fixation $[8,9]$, so they suggested that for rectal cancer, endoscopic material rather than operative material should serve as the primary material for IHC test [9].

However, these were retrospective researches collected from histopathology electronic database and didn't consider inter-observers' variances across different pathologists. The purpose of this study was to evaluate the consistency of endoscopic and surgical specimens for MMRP detection between different pathologists, and analyze factors associated with the variations.

In this study, we asked two pathologists to diagnose 4 MMRP both on endoscopic biopsies and surgical materials in 51 CRC patients respectively and found that among the paired MMRP, 804 (98.5\%) pairs showed concordant IHC stains between two 
pathologists, or between endoscopic and operative material stains, the agreement was almost perfect for MSH6 and PMS2.

Five patients showed inconsistent MMRP, either between different specimens or between two pathologists. In order to identify the clinical, pathologic, and molecular features related to inter-observers' variations in MMRP detection in CRC patients, patients were divided into two groups according to the consistency of MMRP between two specimens: consistent group and inconsistent group. We believe that the issues exist in endoscopic specimens are biopsy samples represent only a small proportion of a tumor and may erroneously classify the MMR status by virtue of inadequate sampling. Clinical and pathological characteristics analysis verified our hypothesis: the consistence of endoscopy biopsy was higher when the number of biopsy debris was more than 4 pieces. In previous studies, generous tumor sampling at the time of biopsy was suggested to reduce the risk of sampling limitations $[11,22,23]$. In this study, more than 4 biopsy pieces were suggested to ensure the accuracy of MMRP detection for the first time. Besides, CRC patients with TNM III-IV stage showed high consistency of MMRP detection due to fewer adenomas in advanced CRC[24], which confirmed the previous conclusion that endoscopic specimens are feasible for the diagnosis of MMRP in advanced CRC patients.

In this research, single-blind method was used to analyze inter-observers' consistence between two pathologists for MMRP detection on endoscopy and surgical specimens for the first time. However, several limitations need to be considered in interpreting our findings. First, measurement errors were inevitable because of the small sample size. Second, although it was a single blind study, clinicopathological data was collected from electronic medical records, selection biases need to be considered. By increasing the number of patients, selection bias and measurement errors were expected to limited.

\section{Conclusions}

In summary, this study showed that inter-observers' variations should be taken account when test mismatch repair proteins on colorectal cancers. Generous endoscopic biopsies could improve the accuracy of endoscopic biopsy for MMRP detection.

The authors declare no conflict of interest.

\section{References}

1. Gil-Raga M, Jantus-Lewintre E, Gallach S, et al. Molecular subtypes in early colorectal cancer associated with clinical fea- tures and patient prognosis. Clinical \& Translational Oncology: Official Publication of the Federation of Spanish Oncology Societies and of the National Cancer Institute of Mexico 2018; 20: 1422-1429.

2. Siegel RL, Miller KD, Fedewa SA, et al. Colorectal cancer statistics, 2017. CA Cancer J Clin 2017; 67: 177-193.

3. Migliore L, Migheli F, Spisni R, et al. Genetics, cytogenetics, and epigenetics of colorectal cancer. J Biomed Biotechnol 2011; 2011: 79236.

4. Laporte GA, Leguisamo NM, Kalil AN, et al. Clinical importance of DNA repair in sporadic colorectal cancer. Crit Rev Oncol Hematol 2018; 126: 168-185.

5. Sinicrope FA, Foster NR, Thibodeau SN, et al. DNA mismatch repair status and colon cancer recurrence and survival in clinical trials of 5-fluorouracil-based adjuvant therapy. J Natl Cancer Inst 2011; 103: 863-875.

6. Amin MB, Greene FL, Edge SB, et al. The Eighth Edition AJCC Cancer Staging Manual: Continuing to build a bridge from a population-based to a more "personalized" approach to cancer staging. CA Cancer J Clin 2017; 67: 93-99.

7. Yoon YS, Yu CS, Kim TW, et al. Mismatch repair status in sporadic colorectal cancer: immunohistochemistry and microsatellite instability analyses. J Gastroenterol Hepatol 2011; 26: $1733-1739$.

8. Vilkin A, Leibovici-Weissman Y, Halpern M, et al. Immunohistochemistry staining for mismatch repair proteins: the endoscopic biopsy material provides useful and coherent results. Hum Pathol 2015; 46: 1705-1711.

9. Vilkin A, Halpern M, Morgenstern S, et al. How reliable is immunohistochemical staining for DNA mismatch repair proteins performed after neoadjuvant chemoradiation? Hum Pathol 2014; 45: 2029-2036.

10. O'Brien O, Ryan E, Creavin B, et al. Correlation of immunohistochemical mismatch repair protein status between colorectal carcinoma endoscopic biopsy and resection specimens. J Clin Pathol 2018; 71: 631-636.

11. Kumarasinghe AP, de Boer B, Bateman AC, et al. DNA mismatch repair enzyme immunohistochemistry in colorectal cancer: a comparison of biopsy and resection material. Pathology 2010; 42: 414-420.

12. Shia J, Stadler Z, Weiser MR, et al. Immunohistochemical staining for DNA mismatch repair proteins in intestinal tract carcinoma: how reliable are biopsy samples? Am J Surg Pathol 2011; 35: 447-454.

13. Shia J, Tang LH, Vakiani E, et al. Immunohistochemistry as first-line screening for detecting colorectal cancer patients at risk for hereditary nonpolyposis colorectal cancer syndrome: a 2-antibody panel may be as predictive as a 4-antibody panel. Am J Surg Pathol 2009; 33: 1639-1645.

14. Barrow E, Jagger E, Brierley J, et al. Semiquantitative assessment of immunohistochemistry for mismatch repair proteins in Lynch syndrome. Histopathology 2010; 56: 331-344.

15. Landis JR, Koch GG. The measurement of observer agreement for categorical data. Biometrics 1977; 33: 159-174.

16. Cappellesso R, Lo Mele M, Munari G, et al. Molecular characterization of "sessile serrated" adenoma to carcinoma transition in six early colorectal cancers. Pathology, research and practice 2019; 215: 957-962.

17. Soong TR, Nayor J, Stachler MD, et al. Clinicopathologic and genetic characteristics of interval colorectal carcinomas favor origin from missed or incompletely excised precursors. Modern Pathol 2019; 32: 666-674.

18. Li GM. Mechanisms and functions of DNA mismatch repair. Cell Res 2008; 18: 85-98.

19. Hewish M, Lord CJ, Martin SA, et al. Mismatch repair deficient colorectal cancer in the era of personalized treatment. Nat Rev Clin Oncol 2010; 7: 197-208. 
20. Sepulveda AR, Hamilton SR, Allegra CJ, et al. Molecular Biomarkers for the Evaluation of Colorectal Cancer: Guideline From the American Society for Clinical Pathology, College of American Pathologists, Association for Molecular Pathology, and the American Society of Clinical Oncology. J Clin Oncol 2017; 35: 1453-1486.

21. Ryan E, Sheahan K, Creavin B, et al. The current value of determining the mismatch repair status of colorectal cancer: A rationale for routine testing. Crit Rev Oncol Hematol 2017; 116: 38-57.

22. Overbeek LI, Ligtenberg MJ, Willems RW, et al. Interpretation of immunohistochemistry for mismatch repair proteins is only reliable in a specialized setting. Am J Surg Pathol 2008; 32: 1246-1251.

23. Funkhouser WK Jr., Lubin IM, Monzon FA, et al. Relevance, pathogenesis, and testing algorithm for mismatch repair-defective colorectal carcinomas: a report of the association for molecular pathology. J Mol Diagn 2012; 14: 91-103.

24. Adan Merino L, Mercedes AM, Jose BA, et al. Factors related to colorectal cancer in advanced adenomas and serrated polyps: a further step toward individualized surveillance. Eur J Gastroenterol Hepatol 2018; 30: 1337-1343.

\section{Address for correspondence}

\section{Shutian Zhang}

Department of Gastroenterology

Beijing Friendship Hospital

Capital Medical University

No. 95, Yong'an Road

Xicheng District

Beijing, 100050 China

e-mail: zhangst@ccmu.edu

Jing Wu

e-mail: wujing36@163.com 